\title{
Is suicidal ideation always a sign of a psychiatric disorder?
}

$\underline{\text { Hugo Canas-Simiãoa }^{*} \text {; Filipa Viegas }}{ }^{\mathrm{b}}$; Rui Ferreira Carvalho ${ }^{c}$; Nuno Rodrigues ${ }^{\mathrm{d}}$; Sara Vilas Boas Garciad; Nuno de Moura ${ }^{\mathrm{a}}$

CENTRO HOSPITALAR D

ISBOA OCIDENTAL, EPE,

CENTRO HOSPITALAR
LISBOA NORTE. EPE
A

No Finantial Interest
hugo.simiao@gmail.com

a Department of Psychiatry and Mental Health, Centro Hospitalar de Lisboa Ocidental, Lisbon, Portugal

b Psychiatry Department, Hospital Prof. Doutor Fernando Fonseca, Amadora, Portugal

c Child and Adolescence Psychiatry Department, Centro Hospitalar de Lisboa Norte, Lisbon, Portugal d Centro Hospitalar Psiquiátrico de Lisboa, Lisbon, Portugal

\section{Introduction and Objectives}

Undoubtedly, mental illness has a higher probability of suicide, being one of the most important predictive factors ${ }^{1}$; however, according to some authors, not all suicidal people are mentally ill ${ }^{2}$. These defend that the suicidal act is constituted of a pathological idea in the majority of times, but can on the other hand come from a deliberate choice of a person without mental pathology 3 .

We report a case that raises a controversial discussion on whether suicidal ideation might be a symptom of a psychiatric disorder or part of rational thinking.

\section{Methods}

The patient underwent a fully structured psychiatric interview and we extracted relevant information from the patient's clinical record after obtaining informed consent. We also performed a nonsystematic review of the literature using the following terms in the Pubmed database: "rational thinking", "suicide" and "psychiatric disorder".

In this clinical case, one of the questions that arises is whether the patient's decision is considered deliberate, authentic, free of mental disturbance. The focus is on whether suicide is a voluntary decision when it is driven by psychological pain with full appreciation of the possible benefits, risks and consequences and not by a diagnosed mental illness.

According to some authors, the relationship between suicide and mental illness is confusing since the latter is not always clearly distinguishable from reactive suffering ${ }^{2,4}$. Others describe suicidal ideation as outside the scope of psychopathology; as

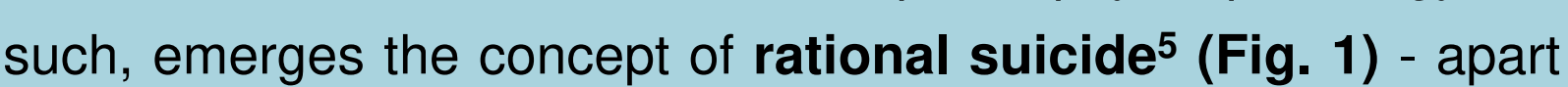
from meeting such criteria very rarely, these criteria are difficult for a clinician to assess.

\section{Clinical Case and Discussion}

A 40-year-old man, with no relevant medical history or prior contact with psychiatry was found ready to jump off a bridge with a suicide note by his side.

He had a long history of a gambling disorder that led him to committing several robberies, unemployment, isolation and rejection from all of his friends and family. At the time of his suicide attempt, he was unemployed, homeless, unable to get out of Portugal and the police was looking out for him for his crimes.

On examination, he was calm and collaborative, keeping his suicidal ideation, but no other psychopathology was found, including signs or symptoms of depression or psychosis.

However, he still accepted to be medicated and to be admitted to a Psychiatry Inpatient Unit and, a week after, he was discharged without death thoughts and referenced to a specialized unit for gambling disorder.

Most current guidelines for managing suicidal ideation and behavior are based on the view that suicide is driven by a mental disorder, not including issues such as the ability for informed and rational decision-making processes regarding suicide ${ }^{4}$. This issue raises difficulties in the management of these patients by clinicians, who on one hand must understand and treat any suffering, but on the other must be sensitive to the person's motivation and autonomy.

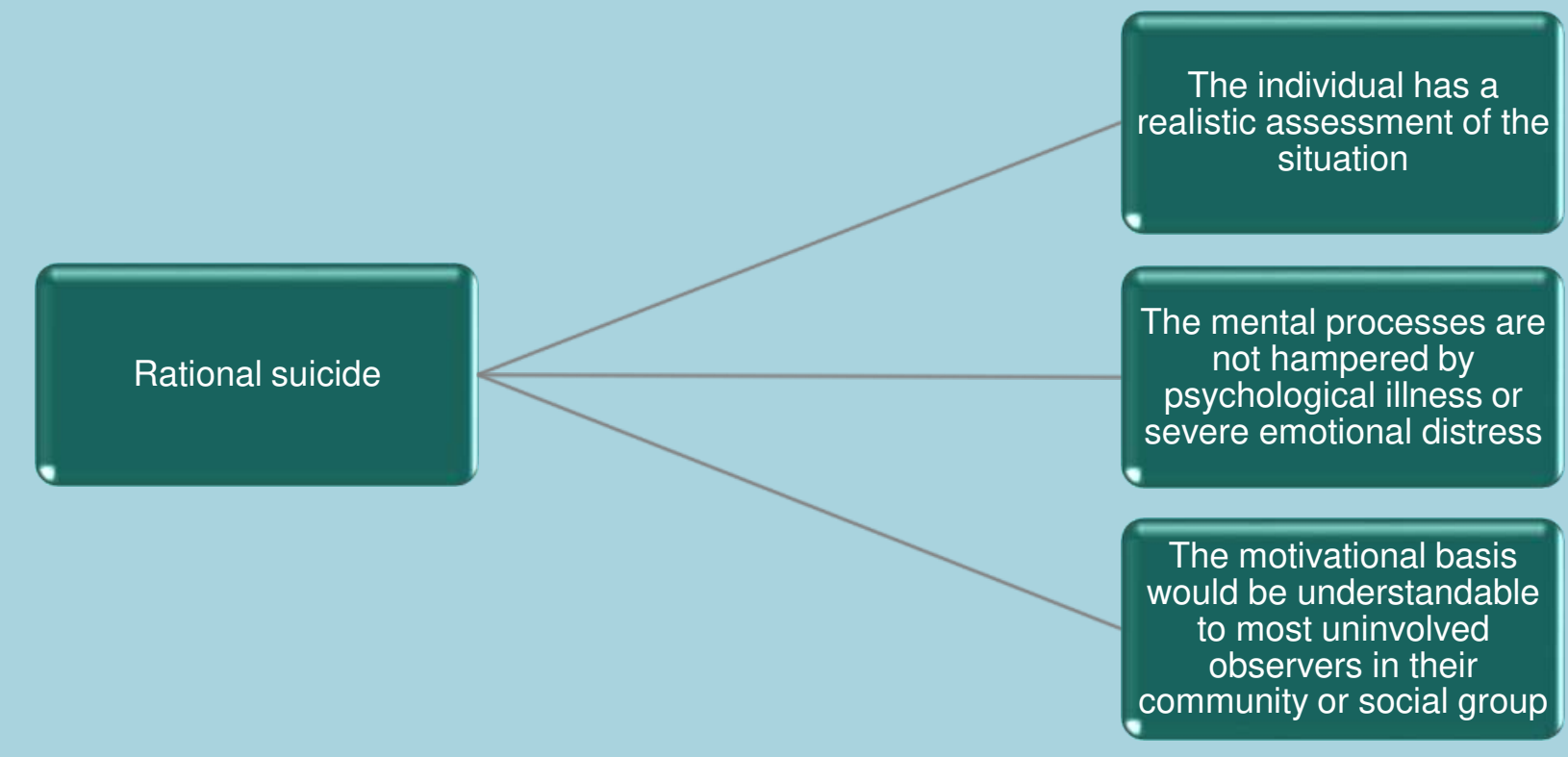

Fig. 1 Rational suicide according to Siegel K. ${ }^{5}$

\section{Conclusions}

$\rightarrow$ The approach to suicidal thoughts should involve multiple domains, focusing on the exploration and treatment of psychopathology, but also consider the multiple related issues (philosophical, ethical, legal, spiritual, among others), including the concept of rational suicide 4 . $\rightarrow$ Since death is irreversible, it may be important to delay action to assess the patient's decision-making ability to understand, diagnose and treat mental disorders, pain, and other forms of suffering that are often treatable and influence the patient's decision 6 .

\section{REFERENCES}

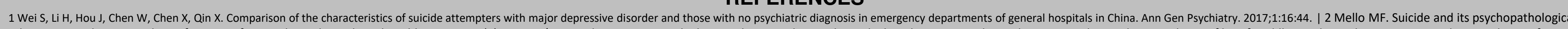

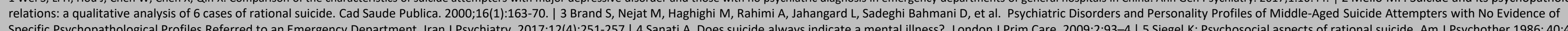

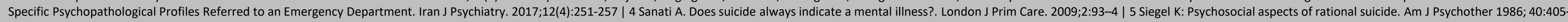
418 | 6 Ho AO. Suicide: rationality and responsibility for life. Can J Psychiatry. 2014; 59(3):141-7 